\title{
Study on frictional behavior of carbon nanotube thin films with respect to surface condition
}

\author{
Youn-Hoo HWANG, Byung-Soo MYUNG, Hyun-Joon KIM* \\ Department of Precision Mechanical Engineering, Kyungpook National University, 2559, Gyeongsang-daero, Sangju, Republic of Korea \\ Received: 16 May 2017 / Revised: 03 July 2017 / Accepted: 04 July 2017 \\ (C) The author(s) 2017. This article is published with open access at Springerlink.com
}

\begin{abstract}
In this work, tribological characteristics of thin films composed of entangled carbon nanotubes (CNTs) were investigated. The surface roughness of CNT thin films fabricated via a dip-coating process was controlled by squeezing during the process with an applied normal force ranging from 0 to $5 \mathrm{kgf}$. Raman spectra and scanning electron microscopy (SEM) images of the thin films were obtained to estimate the influence of the squeezing process on the crystallinity of the CNTs. The analysis revealed that squeezing could reduce surface roughness, while preserving the crystallinity of the CNTs. Moreover, the surface energy of the cover glass used to press the CNT thin film was found to be the critical factor controlling surface roughness. A micro-tribometer and macro-tribometer were used to assess the tribological characteristics of the CNT thin film. The results of the tribotest exhibited a correlation between the friction coefficient and surface roughness. Dramatic changes in friction coefficient could be observed in the micro-tribotest, while changes in friction coefficient in the macro-tribotest were not significant.
\end{abstract}

Keywords: carbon nanotubes; friction; surface roughness; surface energy; squeezing process; UV irradiation

\section{Introduction}

Carbon nanotubes (CNTs) have attracted significant interest for decades because of their superb mechanical, electrical, and chemical characteristics, such as high tensile strength and elastic modulus along the longitudinal direction, and good elastic and thermal conductivity properties [1-4]. Based on these unique properties, various CNT applications have been suggested, such as probe tips for atomic force microscopy (AFM) and scanning tunneling microscopy (STM) $[5,6]$, rotational actuators [7], field emission devices [8-10], electric motor brushes [11, 12], and chemical sensors $[13,14]$. Owing to their small dimensions, CNTs are regarded as suitable materials for exploitation in several precision industries such as semi-conductors, batteries, and display devices $[15,16]$. Some researchers have also focused on the ability of the outstanding mechanical properties of CNTS to improve durability and tribological characteristics. There have been several attempts to exploit CNTs in reinforcing polymers $[17,18]$ or metal composites [19-21]. The utilization of CNTs as additives in lubricants has also been considered by several researchers [22, 23].

Since CNTs exhibit lubricious characteristics and outstanding wear resistance, numerous studies have investigated the fundamental behavior of CNTs for tribological applications. Falvo et al. [24] conducted an experiment to manipulate a single CNT on a mica surface. In the study, they discovered stick-slip motion of the CNT and investigated shear stress at the interface between the CNT and the mica surface. Following the study, Falvo et al. [25] also revealed that sliding and rolling of a CNT are attributed to commensurability of nano-scale contact, suggesting the importance of CNT alignment on tribological characteristics. Kim and Kim [26] studied the frictional behavior of CNTs through molecular dynamics simulation, discovering

* Corresponding author: Hyun-Joon KIM, E-mail: hjoonkim@knu.ac.kr 
that the frictional force of CNTs depends on the interaction between adjacent nanotubes, $\mathrm{CNT}$ orientation, and elastic deformation.

There have been several attempts to exploit CNTs as a surface coating to improve tribological characteristics. Kim et al. [27] revealed the potential of CNTs to minimize surface wear when CNTs were combined with a noble metal that exhibited low shear strength. In the study, CNTs and Ag were coated on a Si surface to form a composite with different thicknesses. The friction coefficient and wear rate of the composite were examined using a pin-on-reciprocating tribometer under $20 \mathrm{mN}$ of normal force. They emphasized that "anchoring" between Ag and CNTs ensured that the composite rarely wore out, even though the individual mechanical properties of the CNTs and Ag were insufficient. Kinoshita et al. [28] reported that a vertically aligned CNT film exhibits high friction coefficients of up to 2.0. Although they investigated vertically aligned CNT films, which show almost zero adhesion force, an extraordinarily high frictional force was reported. They deduced that bent nanotubes corresponding to relative motion of the counter surface demonstrate high repulsive force for elastic recovery and are the cause of the high frictional force.

Although numerous tribological studies have been conducted over decades, there is still a lack of understanding with regard to the complicated behavior of CNTs under various environmental conditions. In addition, the majority of studies on the tribological characteristics of CNTs have been concerned with composites and lubricant additives. Since a CNT film usually consists of numerous fibers, which are entangled together and have various dimensions, tribological characteristics may vary significantly with environmental conditions. Therefore, intensive study on the tribological behavior of CNT thin films is required to enhance fundamental understanding. In this work, the influence of the surface morphology of CNTs on the frictional behavior of CNT thin films was experimentally investigated at the micro-scale.

\section{Experimental setup}

\subsection{Preparation of CNT thin films}

A soda-lime glass slide (Marienfeld, Germany) was chosen as the CNT thin film substrate as it provides a relatively smooth and uniform surface. The glass slide was carefully rinsed with acetone, ethanol, and deionized (DI) water using an ultrasonicator for $10 \mathrm{~min}$ each to remove any surface contamination. The surface energy of the cleaned glass slide was increased by exposing it to UV light for an hour to strengthen the interface bonding between the CNTs and the glass slide. The water contact angle was measured to confirm the change in surface energy on UV irradiation, as shown in Fig. 1. When the glass slide is irradiated with UV light, the water contact angle is about $9^{\circ}$, while that of the glass slide without $\mathrm{UV}$ treatment is $27^{\circ}$. The CNT thin film was coated on the glass slide by a dip-coating method, as shown in Fig. 2. The glass slide was immersed in a CNT suspension (JEIO Co. Ltd., Republic of Korea), which consisted of $95 \mathrm{wt} \%$ of isopropyl alcohol (IPA), $3 \mathrm{wt} \%$ of multi-walled CNTs, and $2 \mathrm{wt} \%$ of surfactants, for a few tens of seconds and withdrawn from the CNT suspension with a velocity of $0.375 \mathrm{~mm} / \mathrm{s}$. Coating parameters such as withdrawal speed may influence the resulting mechanical characteristics of the thin film. It has been well documented that the withdrawal speed affects film thickness [29]. Therefore, the dip-coating conditions were carefully optimized. The immersion time was optimized to stabilize the

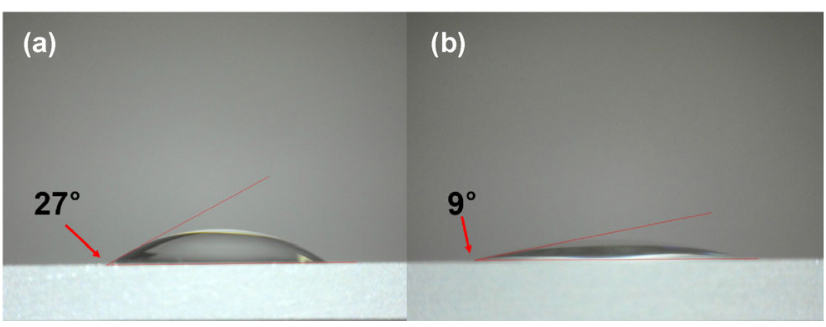

Fig. 1 Water contact angle of (a) untreated and (b) UV-irradiated glass plates.

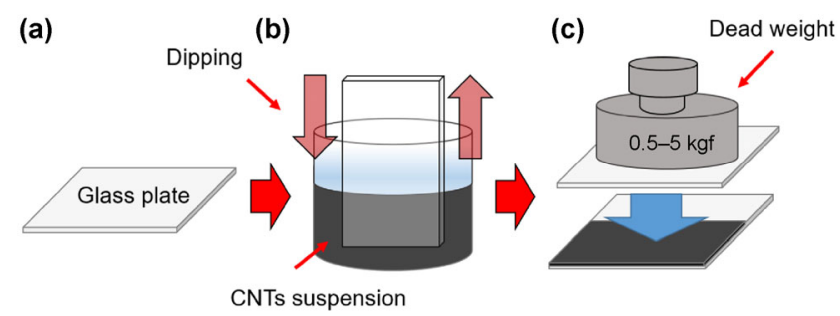

Fig. 2 Schematic of CNT thin film fabrication process: (a) rinsing the borosilicate glass slide, (b) dipping the glass slide into the CNT solution, and (c) squeezing the CNT thin film with a dead weight. 
suspension state. The withdrawal speed was optimized for the target CNT thin film thickness. We measured the thickness of the CNT thin film prepared under the optimized withdrawal rate of $0.375 \mathrm{~mm} / \mathrm{s}$ to be approximately $3 \mu \mathrm{m}$. After the dipping process, both sides of the CNT-coated glass slide were held in dry air to evaporate the IPA. The tribological characteristics of the front face thin film of the CNT-coated glass slide were assessed. The CNT coating on the back face of the glass slide was not removed; rather, the back face was covered with cellophane tape to minimize any uncertainty arising from the CNT thin film on the back face.

Since the CNT thin film consists of an entangled form of numerous nanotubes, occasional surface nanotube protrusions are inevitable, increasing the surface roughness compared to that of the bare glass slide. As the surface roughness of the CNT thin film might influence the tribological characteristics, a squeezing method (shown in Fig. 2(c)) to control surface roughness was suggested in this work. In this process, a glass plate with identical dimensions to the glass substrate was placed over the CNT thin film and a dead weight ranging from 0.5 to $5 \mathrm{kgf}$ was placed on the assembly for $30 \mathrm{~min}$ to apply relatively uniform pressure and maintain stable surface conditions. In addition, UV treatment was applied to the cover-glass plate to investigate the influence of surface energy on the roughness and tribological characteristics of the CNT thin film. It was anticipated that UV-treated cover glass would have a higher surface energy than the glass plate without UV treatment. Therefore, using both untreated and UV-treated cover-glass plates for the squeezing process, the influence of cover-glass surface energy on the CNT thin film could be examined. The effect of the squeezing process on the surface roughness was measured using a laser scanning confocal microscope (VK-X200, Keyence, Japan). Figures 3(a) and 3(b) present 3D images of the surface topography of CNT thin films pressed with respective normal forces of 1 and $5 \mathrm{kgf}$, resulting in high and low surface roughness, respectively. In both cases, the substrate consisted of a glass plate treated with UV light. The exact morphology of the entangled CNTs is not evident in Fig. 3 since the scanned image area is approximately $280 \mathrm{um} \times 200 \mu \mathrm{m}$. Considering that the diameter of the CNTs used in this work is about $100 \mathrm{~nm}$, the image area is too large to describe the fine features of a single nanotube. However, the officially reported microscope resolution $(0.5 \mathrm{~nm}$ in the vertical direction) is regarded as sufficient to measure the surface roughness of the CNT thin film. The images in Fig. 3 confirm that the squeezing process can modify surface roughness effectively.

The change in CNT thin film thickness after pressing with a normal force of $5 \mathrm{kgf}$ was also examined to verify the effect of the squeezing process. Three specimens were tested and the measurement was conducted 3-5 times for each specimen to provide reliable analysis. As a result, it was concluded that there was no significant change in CNT thin film thickness after the squeezing process.

\subsection{Friction test setup and conditions}

In this study, two different tribometers were exploited to measure the frictional force of the CNT thin film. One tribometer was designed to examine the frictional characteristics at the micro-Newton scale, by deflection of thin cantilevers. The other was used to measure

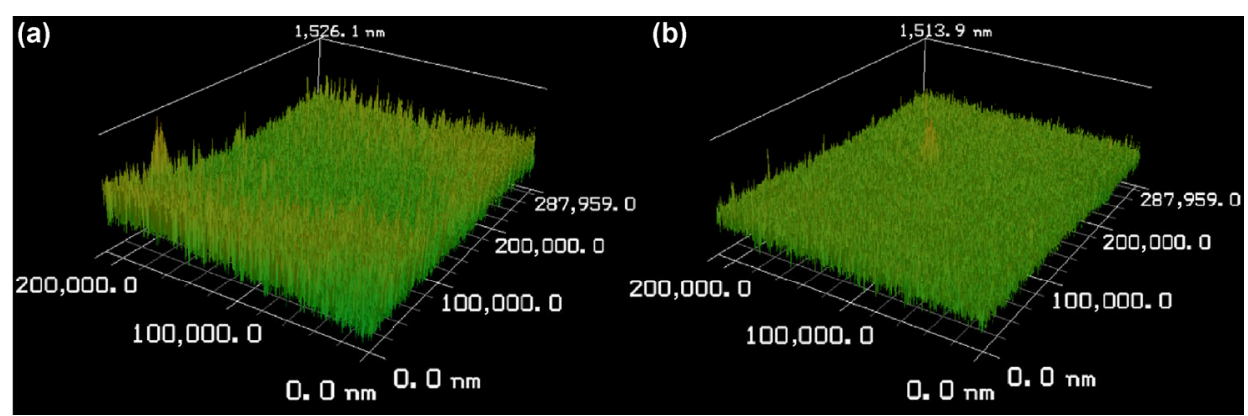

Fig. 3 Surface profiles obtained using a laser scanning confocal microscope of specimens with (a) relatively high surface roughness and (b) low surface roughness. 
frictional force at the milli-Newton scale. For convenience, the former tribometer is referred to as a "micro-tribometer" and the latter is referred to as a "macro-tribometer". The configuration of both tribometers is presented in Fig. 4. The micro-tribometer is a pin-on-disc type apparatus, which was set up with a step motor to precisely control the rotation speed, as shown in Fig. 4(a). To apply an extremely small normal force of about a micro-Newton to the CNTs, a tiny and thin counter specimen consisting of a $3 \times 3 \mathrm{~mm}$ square diced piece of silicon with a thickness of $0.67 \mathrm{~mm}$ and three zirconia spheres with a diameter of $1 \mathrm{~mm}$ was utilized. The spheres, which were in contact with the CNT thin film during the friction test, were bonded to the silicon piece at discrete locations to maintain stable contact conditions. The counter specimen weighed $196 \mu \mathrm{N}(20 \mathrm{mg})$. The CNT thin film-coated glass slide was placed on the circular-plate assembly with step motor and the counter specimen was placed on the CNT thin film. During operation, the glass plate rotated with an angular velocity of $6 \mathrm{rpm}$ and the counter specimen pushed the long cantilever due to frictional force at the interface between its zirconia spheres and the CNT thin film. Two strain gauges were attached to the two flat faces at the end of the cantilever to detect cantilever deflection, and a half-bridge circuit was constructed to quantitatively measure the amount of deflection in voltage. Using a data acquisition system (NI9237, National Instruments, USA), the voltage signal was recorded and converted into a frictional force in Newton units through calibration of the tribometer. Each experiment was performed for $10 \mathrm{~min}$ and, for reliable results, the friction test for each specimen was performed 3 to 5 times.

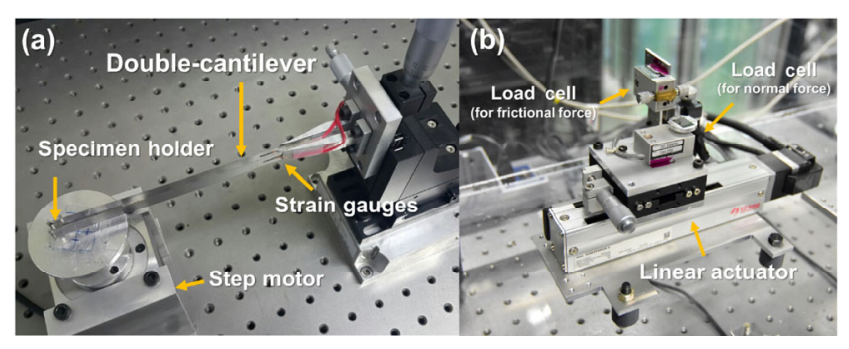

Fig. 4 Construction of tribometer experimental setup: (a) "microtribometer", designed to measure at the micro-Newton scale; (b) "macro-tribometer", designed to measure at the milli-Newton scale.
The macro-tribometer operated as a pin-onreciprocating type of tribotester (Fig. 4(b)). Using the tribometer, the frictional force of CNT thin films under a normal force of $4.9 \mathrm{mN}(0.5 \mathrm{~g})$ was measured. Zirconia spheres with a diameter of $1 \mathrm{~mm}$ were also exploited as the counter surface. These were bonded to the end of the triangular cantilever and the cantilever was assembled with a precision load cell (GSO-30, Transducer Techniques, USA), which was used to measure frictional force at the milli-Newton scale. A linear actuator transported the specimen back and forth, with stroke length, cycle number, and linear velocity of $2 \mathrm{~mm}, 200$ cycles, and $4 \mathrm{~mm} / \mathrm{s}$, respectively. Similarly to the micro-tribotest experiments, the macrotribotest experiments were also conducted 3 to 5 times per specimen for reliability.

The purpose of applying these two tribometers operating at different scales was to evaluate the influence of normal force on the frictional behavior of CNT thin films. Generally, pin-on-disk and pin-onreciprocating type tribometers result in slightly different frictional behavior, owing to discrepancies in their respective sliding motions. In addition, it is possible that each type of tribometer will examine different wear characteristics. However, these tribometers were exploited not to quantitatively compare the friction coefficient between the macro and micro-scales, but to investigate the change in friction coefficient with respect to surface roughness. Therefore, it is believed that exploitation of different types of tribometer has not affected the fundamental analysis.

\section{Experimental results}

\subsection{Surface analysis of CNT thin film with respect to the squeezing process}

Since the dip-coating process is sensitive to various conditions such as temperature, humidity, withdrawal speed, and viscosity of the CNTs suspension, a set of CNT thin film specimens was prepared simultaneously. The temperature and relative humidity during the dip-coating process were $20-22{ }^{\circ} \mathrm{C}$ and $35 \%-40 \%$, respectively. Simultaneous coating of a set of CNT thin film specimens prevented evaporation-induced viscosity changes in the CNT suspension. The set 
consisted of five specimens squeezed by normal forces of $0,0.5,1,2$, and $5 \mathrm{kgf}$. A normal force of $0 \mathrm{kgf}$ implies that this CNT thin film was not squeezed. The five specimens were also prepared simultaneously to minimize uncertainties in the surface condition arising from varying environmental conditions. Assessment of surface roughness was conducted using a laser scanning confocal microscope. For each specimen, the surface roughness of 3 to 5 points was measured and averaged to obtain reliable results. It was revealed that the surface roughness varied with applied squeezing force. When an untreated cover-glass plate was used for the squeezing process, the surface roughness decreased as the squeezing force increased. It was postulated that squeezing the $\mathrm{CNT}$ thin film with a certain weight caused the protruding nanotubes from the relatively smooth surface to deform; thus, the coarse surface topography became smoother. However, when a UV-treated cover-glass plate was used for the squeezing process, the surface roughness was affected differently. In this case, it was observed that the surface roughness of the CNT thin film generally increased as the squeezing force increased. This result can be explained in terms of the surface energy of the glass plate. As shown in Fig. 1, the UV-treated glass plate exhibits a higher surface energy compared to the untreated glass plate. Therefore, it can be deduced that the UV-treated glass plate would exhibit a stronger adhesion force to the CNTs compared to the untreated glass plate. When the CNT thin film was pressed using the untreated glass, each nanotube strand would be elastically deformed. Nevertheless, because of adhesion between nanotubes, the surface morphology of the CNT thin film would not be recovered to its original form after removal of the cover glass. This is regarded as the main mechanism for roughness control in the squeezing process. When the squeezing process was conducted using UV-treated cover glass, the increased adhesion force at the glass surface could result in different CNT thin film behavior. Prior to the release step, the deformation of the nanotube strands would be identical with the previous case. It was anticipated that the difference arises from the release step, in which the cover glass was removed. Because of increased adhesion force between the CNTs and the cover-glass surface, a relatively strong bond between the glass surface and the nanotubes was formed. During the release step, it was assumed that some nanotubes would be pulled off the surface of the CNT thin film due to bonding between the nanotubes and the glass surface, until the bonding force became equal to the sum of the elastic deformation force of the nanotubes and the adhesion force between nanotubes. As the squeezing force was increased, more nanotubes would form contact with the cover glass, strengthening the adhesion force between the glass and the nanotubes. Therefore, when a squeezing force of less than $1 \mathrm{kgf}$ was applied, the surface roughness increased. However, when squeezing forces of 2 and $5 \mathrm{kgf}$ were applied, it was postulated that the decreased surface roughness arising from the pressing step dominated over the increased surface roughness arising from the release step. Therefore, the surface roughness decreased under these conditions. In this regard, the increased surface roughness resulting from the squeezing process with UV-treated glass plate was attributed to the stronger adhesion force pulling off the CNTs attached to the cover-glass surface. Figure 5 displays a schematic representation of this speculative process. The resulting change in surface roughness of the CNT thin film is listed in Table 1. A difference in the surface roughness of unsqueezed CNT thin films is also observed between the untreated and UV-treated glass plates, which exhibit surface roughness values of 177.7 and $80.1 \mathrm{~nm}$, respectively. This is attributed to uncertain factors such as irregular formation of CNT entanglement in the suspension during the coating process. In fact, the surface condition of a CNT thin film formed in this process cannot be similar to any thin film fabricated by a sputtering

(a)
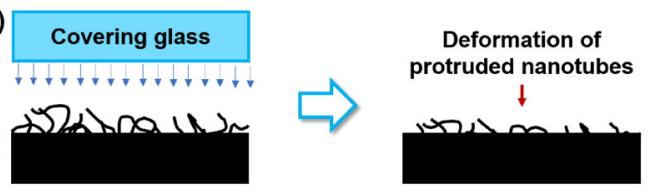

(b)

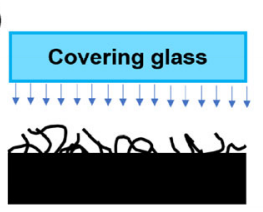

Strong van der Waals force $\uparrow$ Covering glass $1 \pm \pm 1 \pm 11 \pm 1$

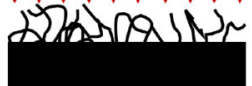

Fig. 5 Schematic of squeezing process reducing surface roughness with (a) untreated cover glass and (b) UV-treated cover glass. 
Table 1 Change in surface roughazness of CNT thin films with varying squeezing force.

\begin{tabular}{ccc}
\hline \multirow{2}{*}{$\begin{array}{c}\text { Squeezing force } \\
(\mathrm{kgf})\end{array}$} & \multicolumn{2}{c}{ Surface roughness (nm) } \\
\cline { 2 - 3 } & $\begin{array}{c}\text { With UV } \\
\text { irradiation }\end{array}$ & $\begin{array}{c}\text { Without UV } \\
\text { irradiation }\end{array}$ \\
\hline 0 & 80.1 & 177.7 \\
0.5 & 88.8 & 171.5 \\
1 & 102.4 & 136.7 \\
2 & 89.3 & 119.3 \\
5 & 73.1 & 109.4 \\
\hline
\end{tabular}

or evaporation method. The CNT thin film formed in this process is not an outcome of atomic level deposition, but rather random agglomeration of nanotube strands. Therefore, regular surface conditions cannot be readily achieved when the CNT thin film is formed using a dip-coating method, while the effect of the squeezing process was clearly confirmed through repeated experiments.

It was assumed that high pressure during the squeezing process might distort the crystal structure of the CNTs. Therefore, the influence of squeezing process on the crystallinity of the CNTs was also estimated using Raman spectroscopy (DXR 2, Thermo Fisher Scientific, USA). For the measurement, a laser operating at $532 \mathrm{~nm}$, with a power of $3.0 \mathrm{~mW}$ and a spot size of $2.0 \mu \mathrm{m}$, was focused on the specimen. Raman spectra at three arbitrary points on each specimen were collected to qualitatively assess the CNT crystallinity. Figure 6 displays the Raman spectra for the CNT thin film squeezed with a normal force of $5 \mathrm{kgf}$ and the unsqueezed CNT thin film. Both spectra show three Raman bands at about 1,350 $\mathrm{cm}^{-1}$ (D band), $1,580 \mathrm{~cm}^{-1}$ ( $\mathrm{G}$ band), and 2,700 $\mathrm{cm}^{-1}$ ( $\mathrm{G}^{\prime}$ band). For ease of viewing, the spectrum obtained from the CNT thin film squeezed with a normal force of $5 \mathrm{kgf}$ is plotted with a vertical offset. As can be seen in Fig. 6, a strong D band associated with defect-induced and dispersive modes is observed. This was attributed to the fact that the nanotubes used in this work were multi-walled and defects occur in the tubes due to unstable crystal structure at the end of the nanotubes or between nanotube walls [30,31]. However, regardless of squeezing process, identical intensities, peak positions, and peak widths were observed. In other words, the squeezing process does not influence the crystal structure of the CNT thin film, but only changes the surface roughness.

Based on a simple calculation, the apparent pressure applied to the CNT thin film when pressed by a dead weight with a normal force of $5 \mathrm{kgf}$ was about $0.1 \mathrm{MPa}$. Considering that the $\mathrm{CNT}$ fracture strength has been reported in some studies to be 50-200 GPa, the stress level during the squeezing process is too small to destroy the CNT crystallinity [32]. Even if there is an occasional stress concentration, the stress would be distributed and minimized through nanotube deformation since the CNT thin film consists of entangled nanotubes, which can readily deform due to a high degree of porosity between the nanotubes, thereby reducing the potential local stress, which could damage CNT crystallinity.

It was expected that the CNT thin film would suffer significant distortion if the high contact pressure caused critical damage. Therefore, the surface morphology with respect to the squeezing process was also investigated using scanning electron microscopy (SEM). Figures 7(a) and 7(b) display the surface condition of the CNT thin film before and after the squeezing process, respectively. Both thin films exhibit a dense entangled structure consisting of nanotubes and no differences

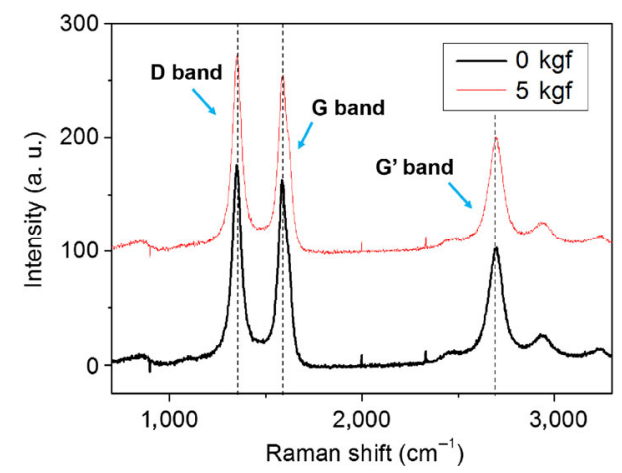

Fig. 6 Raman spectra of CNT thin film with and without squeezing process.

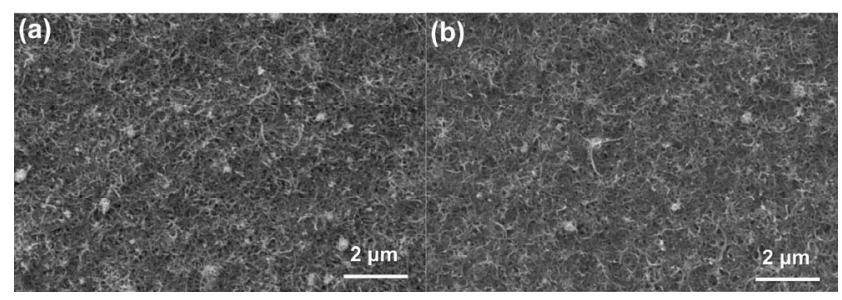

Fig. 7 SEM images of the CNT thin film produced (a) without squeezing process and (b) with a normal force of $5 \mathrm{kgf}$ applied in the squeezing process. 
are observed. Indeed, the squeezing process does not damage the nanotube crystal structure.

\subsection{Frictional force of CNT thin film with respect to surface roughness}

Figure 8 presents frictional force data during a $10 \mathrm{~min}$ micro-tribotest. The figure shows the entire process of the friction test, beginning from the stationary state. At the early stage, zero frictional force is recorded since the step motor is not rotating. Once the step motor is started, a sudden increase in frictional force is observed, followed by a smaller change, which is attributed to run-in behavior during the first approximately $50-100 \mathrm{~s}$. The frictional force then stabilizes rapidly and maintains a relatively steady value during the remainder of the test. Although the frictional force enters a stable stage after approximately 100-200 s, slight fluctuation in friction is observed. This is attributed to both the tribometer construction and the frictional characteristics of the CNT thin film. The micro-tribometer was designed to measure frictional force at the micro-Newton scale. Therefore, the apparatus consisted of a low stiffness cantilever to detect weak frictional signals; the cantilever was highly sensitive to both vibration and change in frictional force. Considering that the surface morphology of the CNT thin film was not perfectly smooth, it could reasonably be estimated that the frictional force at the surface would not be evenly distributed. Because of surface irregularity, a change in friction may arise during measurement and this fluctuation in friction may cause slight vibration of the tribometer. Hence, it was speculated that both factors influenced the measured frictional force signal. However, distinct differences in the average frictional force of the CNT thin film with respect to the squeezing process conditions could be observed, regardless of the outlined variability. Moreover, it was confirmed that the average frictional force did not vary significantly during the test. Therefore, the average of the frictional force was taken as the representative value.

However, since differences in frictional force related to surface roughness were revealed, the relationship between surface roughness and frictional characteristics was evaluated. Figure 9 presents the friction coefficient and surface roughness in relation to the normal force used in the squeezing process with UV-treated glass pate. In the figure, a strong relationship between friction coefficient and surface roughness is observed. As explained in the previous section, an increase of surface roughness even squeezing process was performed can be attributed to the high adhesion force of the cover-glass plate. It is revealed that a change in friction coefficient generally corresponds to a change in surface roughness. However, the squeezed CNT thin film with the lowest surface roughness still exhibits a higher friction coefficient than the unsqueezed CNT thin film. It was postulated that the squeezing process might decrease overall surface waviness, but microscopic protrusion of CNTs was promoted during detachment of the cover glass. Therefore, it is believed that the protruding CNTs disturb the relative motion of the counter surface and thus increase the frictional force. According to Fig. 9, the friction coefficient increases from 0.23 to 0.51 as the surface roughness increases.

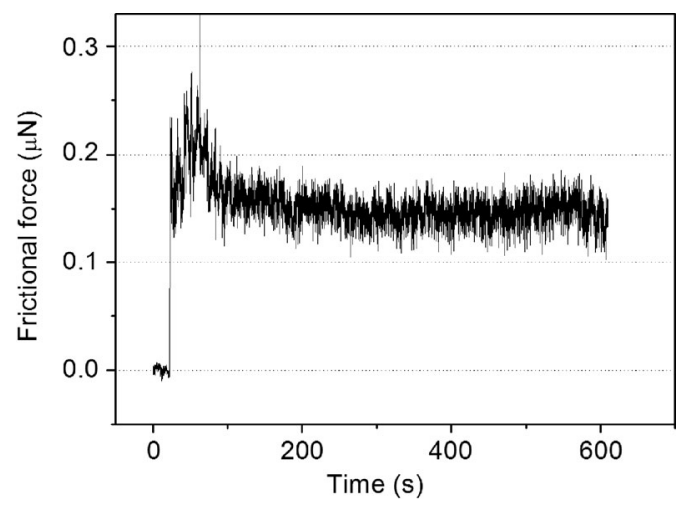

Fig. 8 Frictional behavior of the CNT thin film obtained using the micro-tribometer.

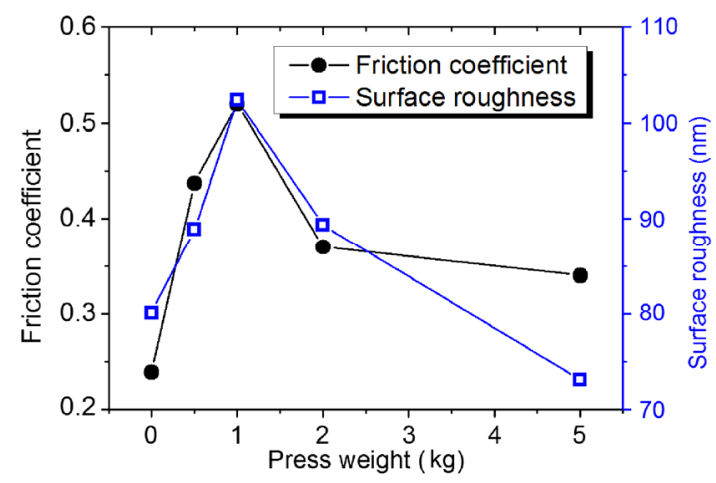

Fig. 9 Micro-tribotest result, which indicates the relationship between surface roughness and the friction coefficient of CNT thin films squeezed using UV-treated glass plate. 
The frictional characteristics of a set of CNT thin films prepared using untreated glass plates were also investigated. In Fig. 10, the relationship between friction coefficient and surface roughness is displayed. It is obvious that the surface roughness decreases as the press weight increases. The trend of change in surface roughness with respect to the normal force applied during the squeezing process is quite different from the previous case for UV-treated glass plates. In Fig. 10, the CNT thin film specimen is squeezed using an untreated glass plate. Since the adhesion force between the glass plate and CNTs is much lower than that between UV-treated glass and CNTs, it is speculated that the squeezing process decreases not only the waviness of the CNT thin film, but also reduces the microscopic irregularity arising from protruding nanotubes. Therefore, it has been confirmed that the friction coefficient continuously decreases from 0.54 to 0.27 as the press weight increases.

The friction coefficient of the CNT thin film with respect to squeezing process at the milli-Newton scale was also evaluated. Since the influence of UV irradiation was revealed through the micro-tribotest, the squeezing process was only performed with untreated cover-glass plate. Similarly to the previous case, the surface roughness of the CNT thin film decreases with increasing press weight, except for the case of $5 \mathrm{kgf}$, as shown in Fig. 11. In this experiment, it is also clear that the friction coefficient is proportional to the surface roughness. The friction coefficient decreases from 0.48 to 0.36 as the press weight increases. Therefore, this result indicates that the surface roughness of the $\mathrm{CNT}$ thin film also influences frictional

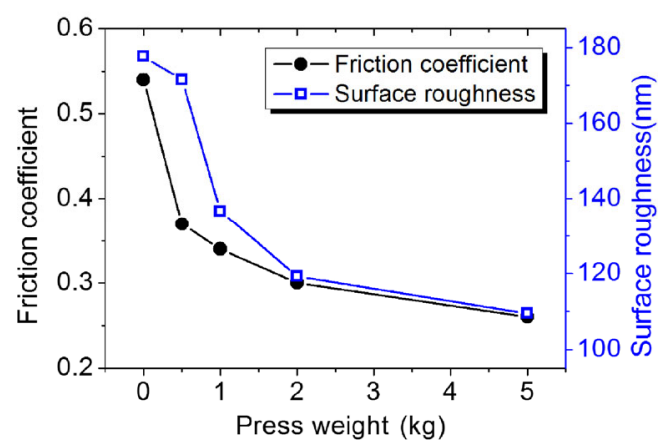

Fig. 10 Micro-tribotest result, which indicates the relationship between surface roughness and the friction coefficient of CNT thin films squeezed using an untreated glass plate.

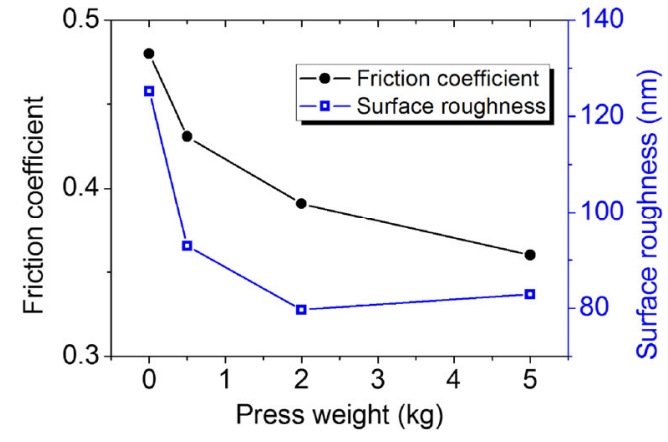

Fig. 11 Macro-tribotest result, which indicates the relationship between surface roughness and the friction coefficient of CNT thin films squeezed using an untreated glass plate.

behavior at the milli-Newton scale. Nevertheless, the change in friction coefficient at the milli-Newton scale is clearly reduced compared to at the micro-Newton scale. In other words, the influence of surface roughness on friction is reduced as the contact pressure increases.

The exception to this observation, at $5 \mathrm{kgf}$, is attributed to complicated factors involved in the surface roughness measurement. It has been assumed that the difference in surface roughness between the specimens pressed by dead weights with normal forces of 2 and $5 \mathrm{kgf}$ is insignificant. However, the examined area in the surface roughness measurement is only approximately $280 \mathrm{um} \times 200 \mu \mathrm{m}$. This area inevitably contains both surface roughness and waviness. Usually, the influence of waviness must be eliminated to obtain surface roughness. However, if there is a complicated wavy structure, the surface waviness would not be completely eliminated. If the surface roughness of two specimens differs sufficiently, imperfectly filtered waviness would not affect the result. In contrast, if two surfaces have similar surface roughness values, then the residual waviness would influence the result. In addition, the roughness data displayed in Fig. 11 are obtained by averaging several measurement points on several specimens. In this process, one relatively large value could be included in the measurement. The exception at $5 \mathrm{kgf}$ is regarded as the outcome of a combination of these factors.

\section{Conclusions}

The frictional characteristics of CNT thin films produced by applying a squeezing method to control 
surface roughness were experimentally investigated. The following points were inferred from the experimental results.

1. The squeezing process could effectively change the surface roughness of the CNT thin film when a cover-glass plate with relatively low surface energy was used. In contrast, cover glass with high surface energy caused the surface condition to deteriorate at a certain press weight. It was speculated that the high surface energy caused the CNTs to partially protrude; these protruded CNTs contributed to the increase in friction.

2. By using Raman spectroscopy and SEM, it was revealed that the squeezing process influenced the surface roughness of the CNT thin film, but did not influence the CNT crystallinity.

3. The friction coefficient of the CNT thin films was generally related to their surface roughness. In particular, a drastic change in friction coefficient (from 0.23 to 0.51 and from 0.27 to 0.54 for UV-treated and untreated cover glass, respectively) with respect to surface roughness was measured in the micro-tribotest, while the macro-tribotest results exhibited only a slight difference in friction coefficient (from 0.36 to 0.48 ) with surface roughness.

4. It was concluded that surface roughness strongly influenced the frictional characteristics at the microNewton scale. Thus, controlling the surface roughness of CNT thin films must be regarded as the most important issue prior to their application in precision systems.

\section{Acknowledgment}

This work was supported by the National Research Foundation of Korea (NRF) grant funded by the Korea government (MSIP) (No. 2015R1C1A1A01053416).

Open Access: The articles published in this journal are distributed under the terms of the Creative Commons Attribution 4.0 International License (http:// creativecommons.org/licenses/by/4.0/), which permits unrestricted use, distribution, and reproduction in any medium, provided you give appropriate credit to the original author(s) and the source, provide a link to the Creative Commons license, and indicate if changes were made.

\section{References}

[1] Fukuda T, Arai F, Dong L. Assembly of nanodevices with carbon nanotubes through nanorobotic manipulation. Proceedings of the IEEE 91(11): 1803-1818 (2003)

[2] Popov V N. Carbon nanotube: Properties and application. Materials Science and Engineering $R$ 43(5): 61-102 (2004)

[3] Dai H. Carbon nanotubes: Synthesis integration, and properties. Acc Chem Res 35(12): 1035-1044 (2002)

[4] Thostenson E T, Ren Z, Chou T W. Advances in the science and technology of carbon nanotubes and their composites: A review. Composites Science and Technology 61(13): 1899-1912 (2001)

[5] Shingaya Y, Nakayama T, Aono M. Caron nanotube tip for scanning tunneling microscopy. Physica B 323(1-4): 153-155 (2002)

[6] Larsen T, Moloni K, Flack F, Eriksson M A, Lagally M G, Black C T. Comparison of wear characteristics of etchedsilicon and carbon nanotube atomic-force microscopy probes. Applied Physics Letters 80(11): 1996-1998 (2002)

[7] Fennimore A M, Yuzvinsky T D, Han W Q, Fuhrer M S, Cumings J, Zettl A. Rotational actuators based on carbon nanotubes. Nature 424: 408-410 (2003)

[8] Doostani N, Darbari S, Mohajerzadeh S, Moravvej-Farshi M K. Fabrication of highly sensitive field emission based pressure sensor, using CNTs grown on micro-machined substrate. Sensors and Actuators A 201: 310-315 (2013)

[9] Tripathi A K, Jain V, Saini K, Lahiri I. Field emission response from multi-walled carbon nanotubes grown on electrochemically engineered copper foil. Materials Chemistry and Physics 187: 39-45 (2017)

[10] Choi B W, Chung D S, Kang J H, Kim H Y, Jin Y W, Han I T, Lee Y H, Jung J E, Lee N S, Park G S, Kim J M. Fully sealed, high-brightness carbon-nanotube field-emission display. Applied Physics Letters 75(20): 3129-3131 (1999)

[11] Pathak S, Cambaz Z G, Kalidindi S R, Swadener. J G, Gogotsi Y. Viscoelasticity and high buckling stress of dense carbon nanotube brushes. Carbon 47(8): 1969-1976 (2009)

[12] Toth G, Maklin J, Halonen N, Palosaari J, Juuti J, Jantunen H, Kordas K, Sawyer W G, Vajtai R, Ajayan P M. Carbon-nanotube-based electrical brush contacts. Advance Materials 21(20): 2054-2058 (2009)

[13] Hu P A, Zhang J, Li L, Wang Z, O’Neill W, Estrela P. Carbon nanostructure-based field-effect transistors for label-free chemical/biological sensors. Sensors 10(5): 5133-5159 (2010) 
[14] Jang Y T, Moon S I, Ahn J H, Lee Y H, Ju B K. A simple approach in fabricating chemical sensor using laterally grown multi-walled carbon nanotubes. Sensors and Actuators $B$ 99(1): 118-122 (2004)

[15] Tian Q, Tian Y, Zhang Z, Yang L, Hirano S. Fabrication of CNT@void@SnO $2 @$ C with tube-in-tube nanostructure as high-performance anode for lithium-ion batteries. Journal Power Sources 291: 173-180 (2015)

[16] Casas C, Li W. A review of application of carbon nanotubes for lithium ion battery anode material. Journal of Power Sources 208: 74-85 (2012)

[17] Kaseem M, Hamad K, Ko Y G. Fabrication and materials properties of polystyrene/carbon nanotube (PS/CNT) composites: A review. European Polymer Journal 79: 36-62 (2016)

[18] Wang L, Song X, Wang T, Wand S, Wang Z, Gao C. Fabrication and characterization of polyethersulfone/carbon nanotubes (PES/CNTs) based mixed matrix membranes (MMMs) for nanofiltration application. Applied Surface Science 330: 118-125 (2015)

[19] Wu Q, Wen M, Chen S, Wu Q. Lamellar-crossing-structured $\mathrm{Ni}(\mathrm{OH})_{2} / \mathrm{CNTs} / \mathrm{Ni}(\mathrm{OH})_{2}$ nanocomposite for electrochemical supercapacitor materials. Journal of Alloys and Compounds 646: 990-997 (2015)

[20] Bastwros M M H, Esawi A M K, Wifi A. Friction and wear behavior of Al-CNT composites. Wear 307(1-2): 164-173 (2013)

[21] Nguyen K C, Ngoc M P, Nguyen M V. Enhanced photocatalytic activity of nanohybrids $\mathrm{TiO}_{2} / \mathrm{CNTs}$ materials. Materials Letters 165: 247-251 (2016)

[22] Chen C S, Chen X H, Xu L S, Yang Z, Li W H. Modification of multi-walled carbon nanotubes with fatty acid and their tribological properties as lubricant additive. Carbon $\mathbf{4 3}(8)$ : 1660-1666 (2005)

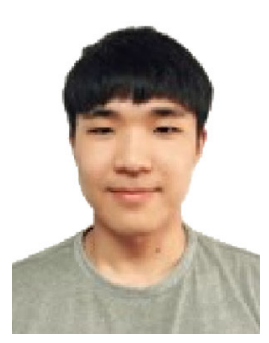

Youn-Hoo HWANG. He received his bachelor degree in Department of Precision Mechanical Engineering in 2017 from Kyungpook National University, Sangju, Republic of
[23] Yu B, Lu Z, Zhou F, Liu W, Liang Y. A novel lubricant additive based on carbon nanotubes for ionic liquids. Materials Letters 62(17-18): 2976-2969 (2008)

[24] Falvo M R, Taylor II R M, Helser A, Chi V, Brooks Jr F P, Washburn S, Superfine R. Nanometre-scale rolling and sliding of carbon nanotubes. Nature 397: 236-238 (1999)

[25] Falvo M R, Steele J, Taylor II R M, Superfine R. Evidence of commensurate contact and rolling motion: AFM manipulation studies of carbon nanotubes on HOPG. Tribology Letters 9: 73-76 (2000)

[26] Kim H J, Kim D E. MD simulation of the frictional behavior of CNTs with respect to orientation. Tribology International 50: 51-56 (2012)

[27] Kim D E, Kim C L, Kim H J. A novel approach to wear reduction of micro-components by synthesis of carbon nanotube-silver composite coating. CIRP Annals-Manufacturing Technology 60: 599-602 (2011)

[28] Kinoshita H, Kume I, Tagawa M, Ohmae N. High friction of a vertically aligned carbon-nanotube film in microtribology. Applied Physics Letters 85(14): 2780-2781 (2004)

[29] Yimsiria P, Mackley M R. Spin and dip coating of lightemitting polymer solutions: Matching experiment with modelling. Chemical Engineering Science 61(11): 3496-3505 (2006)

[30] Osswald S, Flahaut E, Ye H, Gogotsi Y. Elimination of D-band in Raman spectra of double-wall carbon nanotubes by oxidation. Chemical Physics Letters 402: 422-427 (2005)

[31] Dresselhaus M S, Dresselhaus G, Jorio A, Souza Filho A G, Saito R. Raman spectroscopy on isolated single wall carbon nanotubes. Carbon 40: 2043-2061 (2002)

[32] Yang L, Greefeld I, Wagner H D. Toughness of carbon nanotubes conforms to classic fracture mechanics. Science Advances 2(2): e1500969 (2016)

Korea. Currently, he is M.S. candidate at Kyungpook National University. His research interests include tribological characteristics of nanomaterial and wear reduction mechanism. 


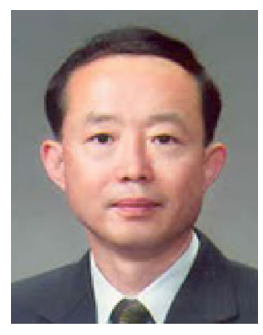

Byung-Soo MYUNG. He received his M.S. and $\mathrm{PhD}$ degrees in mechanical engineering from Chungnam National University, Daejeon, Republic of Korea, in 1986 and 1993

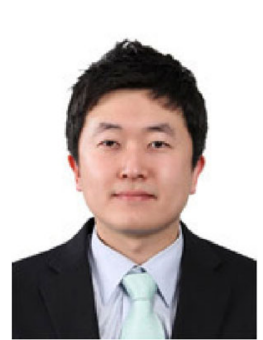

Hyun-Joon KIM. He received bachelor and $\mathrm{PhD}$ degrees in mechanical engineering from Yonsei University, Seoul, Republic of Korea, in 2005 and 2012, respectively. He respectively. He is currently chair of Department of Precision Mechanical Engineering, Kyungpook National University. His research interests are reduction of friction through vibration and contact characteristics during vibration.

joined Department of Precision Mechanical Engineering at Kyungpook National University as assistant professor from 2014. His current interests are AFM based nano-mechanics, micro-nano tribology, and molecular dynamics simulation. 